\title{
GROMOV-HAUSDORFF CONVERGENCE OF QUANTISED INTERVALS
}

\author{
THOMAS GOTFREDSEN, JENS KAAD, AND DAVID KYED
}

\begin{abstract}
The Podleś quantum sphere $S_{q}^{2}$ admits a natural commutative $C^{*}$-subalgebra $I_{q}$ with spectrum $\{0\} \cup\left\{q^{2 k}: k \in \mathbb{N}_{0}\right\}$, which may therefore be considered as a quantised version of a classical interval. We study here the compact quantum metric space structure on $I_{q}$ inherited from the corresponding structure on $S_{q}^{2}$, and provide an explicit formula for the metric induced on the spectrum. Moreover, we show that the resulting metric spaces vary continuously in the deformation parameter $q$ with respect to the Gromov-Hausdorff distance, and that they converge to a classical interval of length $\pi$ as $q$ tends to 1 .
\end{abstract}

\section{INTRODUCTION}

The study of compact quantum metric spaces dates back to the work of Connes [Co89], in which he studied metrics on state spaces of spectral triples. This notion was later formalised in the works of Rieffel [Ri98, Ri99, Ri05, in which the weak $*$-topology on the state space is metrised by the Monge-Kantorovich metric coming from a so-called Lip-norm on a $C^{*}$-algebra (see Section 2 for details). As shown by Rieffel, the classical Gromov-Hausdorff distance admits an analogue, known as quantum Gromov-Hausdorff distance, for compact quantum metric spaces, and this notion was later refined by Latrémolière through his notion of propinquity La16]. Although examples of compact quantum metric spaces are abundant, some of the most basic examples from non-commutative geometry are not well understood from this point of view, and only very recently, Aguilar and Kaad AK18 showed that the Podleś standard sphere $S_{q}^{2}$, introduced as a homogeneous space of Woronowicz' $q$-deformed $S U(2)$ Po87, Wo87, admits a natural compact quantum metric space structure stemming from its non-commutative geometry. More precisely, Aguilar and Kaad show that the Lip-norm arising from the Dirac operator $D_{q}$ of the Dąbrowski-Sitarz spectral triple DS03, does indeed provide a quantum metric structure on $S_{q}^{2}$. The main question left open in AK18] is that of quantum GromovHausdorff convergence of $S_{q}^{2}$ to the classical 2-sphere $S^{2}$ as the deformation parameter tends to 1 . This question seems rather difficult to settl]1, and the aim of the present paper is to show that the Podles sphere $S_{q}^{2}$ contains a natural commutative $C^{*}$-algebra $I_{q}$ for which the corresponding convergence question can be settled, and that the answer supports the more general conjecture that $S_{q}^{2}$ converges to $S^{2}$ as $q$ tends to 1 . The Podleś sphere is generated by a self-adjoint operator $A$ and a non-normal operator $B$ (see Section 2 for precise definitions), and the $C^{*}$-algebra $I_{q}$ is simply the unital $C^{*}$-algebra generated by $A$ inside $S_{q}^{2}$. Since $S_{q}^{2}$ admits a rather accessible representation on $B\left(\ell^{2}\left(\mathbb{N}_{0}\right)\right)$ [Po87, Proposition 4], the spectrum of the self-adjoint generator $A \in S_{q}^{2}$ is easily derivable, and one finds that for $q \in(0,1)$ this is exactly the set

2010 Mathematics Subject Classification. 58B32, 58B34, 46L89, 46L30.

Key words and phrases. Quantum metric spaces, Podleś sphere, Gromov-Hausdorff distance.

${ }^{1}$ Note: building on the results of the present paper, this was very recently settled in AKK21. 


$$
X_{q}=\{0\} \cup\left\{q^{2 k}: k \in \mathbb{N}_{0}\right\},
$$

which can therefore be viewed as a quantised version of a classical interval. The Lip-norm $L_{D_{q}}$ coming from the Dirac operator on $S_{q}^{2}$ therefore, in particular, provides a metric on the state space of $I_{q} \cong C\left(X_{q}\right)$ and embedding $X_{q}$ into the state space of $C\left(X_{q}\right)$ as point-evaluations, we obtain a metric $d_{q}$ on $X_{q}$. Our first main result determines an explicit formula for this metric.

Theorem A. For $q \in(0,1)$, the metric $d_{q}$ on $X_{q}$ is given by the following formula:

$$
d_{q}(x, y):= \begin{cases}\sum_{k=\min \{m, n\}}^{0} \frac{\left(1-q^{2}\right) q^{k}}{\sqrt{1-q^{2(k+1)}}} & \text { if } x=q^{2 n} \text { and } y=q^{2 m} \text { with } n \neq m \\ \sum_{k=n}^{\infty} \frac{\left(1-q^{2}\right) q^{k}}{\sqrt{1-q^{2(k+1)}}} & \text { if } x=q^{2 n} \text { and } y=0 \text { or } x=0 \text { and } y=q^{2 n} .\end{cases}
$$

When $q=1$, the spectrum of the operator $A$ becomes $X_{1}:=[0,1]$ and in Section 3.1 we will show that when $X_{1}$ is equipped with the metric $d_{1}$ inherited from the classical 2-sphere $S^{2}$, then the space $\left(X_{1}, d_{1}\right)$ becomes isometrically isomorphic to $\left[-\frac{\pi}{2}, \frac{\pi}{2}\right]$ with its standard Euclidian metric. Our second main theorem therefore confirms that the quantised intervals do indeed converge to the appropriate classical interval as the deformation parameter tends to 1:

Theorem B. The metric spaces $\left(X_{q}, d_{q}\right)$ vary continuously with respect to the GromovHausdorff distance in the deformation parameter $q \in(0,1)$ and converge to the interval $\left[-\frac{\pi}{2}, \frac{\pi}{2}\right]$ with its standard metric as $q$ tends to 1.

On the class of commutative compact quantum metric spaces, convergence in both Latrémolière's propinquity La16 and Rieffel's quantum Gromov-Hausdorff distance Ri04 is implied by convergence in classical Gromov-Hausdorff distance (see Remark 3.7) and Theorem $\mathrm{B}$ therefore settles all the natural convergence question for the algebras $I_{q} \cong C\left(X_{q}\right)$.

The paper is structured as follows: In the first part we introduce the basic definitions concerning quantum metric spaces, Gromov-Hausdorff distance, $S U_{q}(2)$ and the standard Podleś sphere and the associated Dąbrowski-Sitarz spectral triple. In the second part we first give a description of $I_{q}$ in the continuum case, i.e. when $q=1$, followed by a thorough treatment of the quantised case, where $S U(2)$ is deformed by a parameter $q \in(0,1)$. For this we provide a detailed treatment of the metric $d_{q}$, on $X_{q}$ and its Lipschitz semi-norm from which we can prove Theorem $\mathrm{A}$, and finally we use this to prove Theorem B.

Acknowledgments. The authors gratefully acknowledge the financial support from the Independent Research Fund Denmark through grant no. 7014-00145B and grant no. 9040-00107B. We are also grateful for the comments and suggestions provided by the anonymous referee.

Standing conventions. The semi-norms appearing in this text are defined everywhere on unital $C^{*}$-algebras and may take the value infinity. 


\section{Preliminaries}

2.1. Quantum metric spaces. We begin this section by recalling some basic facts about metric spaces. Let $(X, d)$ be a compact metric space. The Lipschitz semi-norm, $L_{d}: C(X) \rightarrow$ $[0, \infty]$, on $C(X)$ is defined by the formula

$$
L_{d}(f):=\sup \left\{\frac{|f(x)-f(y)|}{d(x, y)}: x \neq y\right\} ; \quad f \in C(X) .
$$

A continuous function $f: X \rightarrow \mathbb{C}$ is then said to be a Lipschitz function when $L_{d}(f)<\infty$ and in this case $L_{d}(f)$ agrees with the Lipschitz constant. The Lipschitz functions on $X$ form a $*$-subalgebra which we denote by $C_{\text {Lip }}(X) \subset C(X)$. Given subsets $A, B \subset X$, their Hausdorff-distance is defined as

$$
\operatorname{dist}_{H}^{d}(A, B):=\inf \{r \geqslant 0 \mid A \subset \mathbb{B}(B, r) \text { and } B \subset \mathbb{B}(A, r)\},
$$

where $\mathbb{B}(A, r)$ denotes the set $\{x \in X: \exists a \in A: d(x, a)<r\}$. For two metric spaces $\left(X, d_{X}\right),\left(Y, d_{Y}\right)$, their Gromov-Hausdorff distance is defined as

$$
\operatorname{dist}_{G H}(X, Y)=\inf \left\{\operatorname{dist}_{H}^{d_{Z}}\left(\iota_{X}(X), \iota_{Y}(Y)\right\},\right.
$$

where the infimum ranges over all metric spaces $\left(Z, d_{Z}\right)$ and all isometric embeddings $\iota_{X}: X \rightarrow$ $Z$ and $\iota_{Y}: Y \rightarrow Z$. Next, we will recall the relevant definitions for quantum metric spaces.

Definition 2.1 ([Ri98, Ri99, Ri05]). Let $A$ be a unital $C^{*}$-algebra, and let $L: A \rightarrow[0, \infty]$ be a semi-norm. We say that $(A, L)$ is a compact quantum metric space, and that $L$ is a Lip-norm, if the following conditions are satisfied:

(1) $\operatorname{Dom}(L):=\{a \in A: L(a)<\infty\}$ is dense in $A$;

(2) $L$ is $*$-invariant and lower semi-continuous on $A$;

(3) $\operatorname{ker}(L):=\{a \in A: L(a)=0\}=\mathbb{C} 1_{A}$;

(4) The Monge-Kantorovich metric on the state space $S(A)$ of $A$, given by

$$
\operatorname{mk}_{L}(\mu, \nu):=\sup \{|\mu(a)-\nu(a)|: a \in A, L(a) \leqslant 1\}, \quad \text { for } \mu, \nu \in S(A)
$$

metrises the weak $*$-topology.

The model example for a compact quantum metric space is, unsurprisingly, $\left(C(X), L_{d}\right)$ where $(X, d)$ is a compact metric space. In this case it is a well-known fact that the MongeKantorovich metric recaptures the metric $d$ on $X$ when the latter is viewed as a subset of the state space of $C(X)$ :

$$
d(x, y)=\sup \left\{|f(x)-f(y)|: f \in C(X), L_{d}(f) \leqslant 1\right\} .
$$

Another interesting class of examples, which dates back to the work of Connes [Co89], comes from certain spectral triples: the setting is thus that of a separable Hilbert space $H$ with a selfadjoint densely defined operator $D: \operatorname{Dom}(D) \rightarrow H$, and a unital $C^{*}$-algebra $A$ represented on $H$ via a $*$-homomorphism $\rho: A \rightarrow B(H)$. Then one can define the Lipschitz algebra $\operatorname{Lip}_{D}(A)$, to consist of all elements $x \in A$ which preserve $\operatorname{Dom}(D)$, and for which $[D, \rho(x)]: \operatorname{Dom}(D) \rightarrow$ $H$ admits a bounded extension to $H$, which will be denoted by $\partial(x) \in B(H)$. Clearly, $\operatorname{Lip}_{D}(A) \subset A$ is a $*$-subalgebra and it follows from the definition of a spectral triple that $\operatorname{Lip}_{D}(A) \subset A$ is norm-dense. From the spectral triple $(A, H, D)$, we also obtain a semi-norm as follows:

Definition 2.2. Define $L_{D}: A \rightarrow[0, \infty]$ by the formula

$$
L_{D}(x):=\sup \left\{\left|\left\langle\xi, \rho\left(x^{*}\right) D \eta\right\rangle-\langle\rho(x) D \xi, \eta\rangle\right|: \xi, \eta \in \operatorname{Dom}(D),\|\xi\|=\|\eta\|=1\right\} .
$$


A first result says that $x \in \operatorname{Lip}_{D}(A)$ exactly when $L_{D}(x)$ is finite, and in this case $L_{D}(x)=\|\partial(x)\|$, see e.g. AK18, Lemma 2.3]. Moreover, $L_{D}: A \rightarrow[0, \infty]$ is lower semicontinuous and $*$-invariant, see [Ri99, Proposition 3.7]. The above construction does in general not yield a quantum metric space, but due to the work of Rieffel, there are tools available for verifying whether or not this is the case (see for instance [Ri98, Theorem 1.8]).

Quantum analogues of the Gromov-Hausdorff distance have been defined by Rieffel and Latrémolière, and we refer the reader to [Ri04, La16] for concrete definitions. For our purposes, it suffices to know that when the compact quantum metric spaces in question are of the form $\left(C(X), L_{d}\right)$, then both analogues are dominated by the classical Gromov-Hausdorff distance, see Remark 3.7.

2.2. The Standard Podleś Sphere. The central object of interest in this paper is the standard Podleś quantum sphere, which is defined as a particular $C^{*}$-subalgebra of Woronowicz' Wo87 quantum group $S U_{q}(2)$ as given below. Fix $q \in(0,1]$, and let $S U_{q}(2)$ denote the universal unital $C^{*}$-algebra with generators $a$ and $b$ defined such that the following relations are satisfied:

$$
\begin{aligned}
b a=q a b, \quad b^{*} a & =q a b^{*}, \quad b b^{*}=b^{*} b \\
a^{*} a+q^{2} b b^{*}=1 & =a a^{*}+b b^{*} .
\end{aligned}
$$

We denote the unital $*$-subalgebra generated by $a$ and $b$ by $\mathcal{O}\left(S U_{q}(2)\right)$, and by $\mathcal{O}\left(S_{q}^{2}\right)$ the unital $*$-subalgebra of $\mathcal{O}\left(S U_{q}(2)\right)$ generated by the elements

$$
A:=b^{*} b \quad \text { and } \quad B:=a b^{*} .
$$

The standard Podleś quantum sphere, $S_{q}^{2}$, is defined as the norm-closure of $\mathcal{O}\left(S_{q}^{2}\right) \subset S U_{q}(2)$ [P087. We remark that from the defining relations of $S U_{q}(2)$ we obtain a similar set of relations for $A$ and $B$ :

$$
\begin{array}{cl}
A B=q^{2} B A, & A=A^{*} \\
B B^{*}=q^{-2} A(1-A), & B^{*} B=A\left(1-q^{2} A\right) .
\end{array}
$$

The $C^{*}$-algebra $S U_{q}(2)$ comes equipped with a natural faithful state, called the Haar state, which we denote by $h: S U_{q}(2) \rightarrow \mathbb{C}$, see e.g. [KS97, Section 11.3.2]. We let $L^{2}\left(S U_{q}(2)\right)$ denote the separable Hilbert space obtained by applying the GNS-construction to the $C^{*}$ algebra $S U_{q}(2)$ equipped with the Haar state.

From now on, we assume that $q \neq 1$. Define an automorphism $\partial_{k}$ on $\mathcal{O}\left(S U_{q}(2)\right)$ by $\partial_{k}(x)=$ $q^{\frac{1}{2}} x$ if $x \in\{a, b\}$, and $\partial_{k}(x)=q^{-\frac{1}{2}} x$ if $x \in\left\{a^{*}, b^{*}\right\}$, and for each $n \in \mathbb{Z}$, define the vector subspaces

$$
\mathcal{A}_{n}:=\left\{x \in \mathcal{O}\left(S U_{q}(2)\right): \partial_{k}(x)=q^{n / 2} x\right\} \subset \mathcal{O}\left(S U_{q}(2)\right) .
$$

It turns out that $\mathcal{A}_{0}=\mathcal{O}\left(S_{q}^{2}\right)$ and that the algebra structure on $\mathcal{O}\left(S U_{q}(2)\right)$ allows us to consider each $\mathcal{A}_{n}$ as a left module over $\mathcal{O}\left(S_{q}^{2}\right)$. We let $H_{+}$and $H_{-}$denote the separable Hilbert spaces obtained by taking the Hilbert space closures of $\mathcal{A}_{1}$ and $\mathcal{A}_{-1}$ (respectively) when considered as subspaces of $L^{2}\left(S U_{q}(2)\right)$. The GNS-representation of $S U_{q}(2)$ on $L^{2}\left(S U_{q}(2)\right.$ ) (when properly restricted) then provides us with two unital $*$-homomorphisms $\rho_{+}: S_{q}^{2} \rightarrow B\left(H_{+}\right)$and $\rho_{-}$: $S_{q}^{2} \rightarrow B\left(H_{-}\right)$ 
By [DS03] there exists an even spectral triple, $\left(S_{q}^{2}, H_{+} \oplus H_{-}, D_{q}\right)$, where the representation in question is given by the direct sum $\rho: \rho_{+} \oplus \rho_{-}: S_{q}^{2} \rightarrow B\left(H_{+} \oplus H_{-}\right)$. For an explicit construction of the Dirac operator $D_{q}: \operatorname{Dom}\left(D_{q}\right) \rightarrow H_{+} \oplus H_{-}$, we refer to [DS03, NeTu05] or AK18.

For $x \in \operatorname{Lip}_{D_{q}}\left(S_{q}^{2}\right)$, the associated operator $\partial(x)$ (obtained as the closure of $\left.\left[D_{q}, \rho(x)\right]\right)$ takes the form

$$
\left(\begin{array}{cc}
0 & \partial_{2}(x) \\
\partial_{1}(x) & 0
\end{array}\right): H_{+} \oplus H_{-} \rightarrow H_{+} \oplus H_{-},
$$

where $\partial_{1}: \operatorname{Lip}_{D_{q}}\left(S_{q}^{2}\right) \rightarrow B\left(H_{+}, H_{-}\right)$and $\partial_{2}: \operatorname{Lip}_{D_{q}}\left(S_{q}^{2}\right) \rightarrow B\left(H_{-}, H_{+}\right)$are derivations satisfying $\partial_{2}\left(x^{*}\right)=-\partial_{1}(x)^{*}$ (remark in this respect that $B\left(H_{+}, H_{-}\right)$and $B\left(H_{-}, H_{+}\right)$can be considered as bimodules over $S_{q}^{2}$ via the representations $\rho_{+}$and $\left.\rho_{-}\right)$. Consequently the Lipnorm is, for $x \in \operatorname{Lip}_{D_{q}}\left(S_{q}^{2}\right)$, given by

$$
L_{D_{q}}(x)=\max \left\{\left\|\partial_{1}(x)\right\|,\left\|\partial_{1}\left(x^{*}\right)\right\|\right\} .
$$

By [Po87, Proposition 4], $S_{q}^{2}$ admits a faithful representation, $\pi: S_{q}^{2} \rightarrow B\left(\ell^{2}\left(\mathbb{N}_{0}\right)\right)$, defined by

$$
\pi(A)\left(e_{k}\right):=q^{2 k} e_{k}, \quad \pi(B)\left(e_{k}\right)=q^{k} \sqrt{1-q^{2(k+1)}} e_{k+1},
$$

where $e_{k}$ denotes the characteristic function on the point-set $\{k\} \subset \mathbb{N}_{0}$. In fact, this representation even provides a $*$-isomorphism to the unitisation of the compact operators on $\ell^{2}\left(\mathbb{N}_{0}\right)$. Using this representation it is easy to see that the spectrum of the operator $A$ for a specific $q \in(0,1)$ is given by

$$
X_{q}:=\{0\} \cup\left\{q^{2 k}: k \in \mathbb{N}_{0}\right\} .
$$

Hence the indicator functions $\chi_{\left\{q^{2 k}\right\}}: X_{q} \rightarrow\{0,1\}$ are continuous for all $k$. In fact, these indicator functions and the unit generate $C\left(X_{q}\right)$, since any continuous function, $f: X_{q} \rightarrow \mathbb{C}$, can be written as $f(0)+\sum_{k=0}^{\infty}\left(f\left(q^{2 k}\right)-f(0)\right) \cdot \chi_{\left\{q^{2 k}\right\}}$, where $\lim _{k \rightarrow \infty} f\left(q^{2 k}\right)=f(0)$. By [AK18, Theorem 8.3], $\left(S_{q}^{2}, L_{D_{q}}\right)$ is a compact quantum metric space, and consequently so is $I_{q}:=C^{*}(A, 1) \cong C\left(X_{q}\right)$ with the restricted Lip-norm. The compact quantum metric space $\left(I_{q}, L_{D_{q}}\right)$ is our main object of interest in the present paper. As $I_{q}$ is commutative, the Lipnorm $L_{D_{q}}$ defines a genuine metric $d_{q}$ on $X_{q}$ when the latter is considered as a subset of the state space $S\left(S_{q}^{2}\right)$. In order to describe $d_{q}$ explicitly, the following lemma will be key:

Lemma 2.3 ([AK18, Lemma 5.3]). Let $k \in \mathbb{N}_{0}$ and let $q \in(0,1)$. We have that $\chi_{\left\{q^{2 k}\right\}}(A) \in$ $\operatorname{Lip}_{D_{q}}\left(S_{q}^{2}\right)$ and the derivative is given by

$$
\partial_{1}\left(\chi_{\left\{q^{2 k}\right\}}(A)\right)=\frac{1}{q^{2 k}\left(1-q^{2}\right)} \chi_{\left\{q^{2 k}\right\}}(A) \cdot b^{*} a^{*}-\frac{1}{q^{2(k-1)}\left(1-q^{2}\right)} \chi_{\left\{q^{2(k-1)}\right\}}(A) \cdot b^{*} a^{*}
$$

In particular, we obtain that

$$
\partial_{1}(f(A))=\sum_{k=0}^{\infty} \frac{f\left(q^{2 k}\right)-f\left(q^{2(k+1)}\right)}{q^{2 k}\left(1-q^{2}\right)} \chi_{\left\{q^{2 k}\right\}}(A) \cdot b^{*} a^{*}
$$

for every $f \in \operatorname{span}_{\mathbb{C}}\left\{\chi_{\left\{q^{2 k}\right\}}: k \in \mathbb{N}_{0}\right\}$.

Remark 2.4. The formula in (2) for $\partial_{1}(f(A))$ is related to the notion of $q$-differentiation from $q$-calculus. Indeed, the $q^{2}$-differentiation of $f \in \operatorname{span}_{\mathbb{C}}\left\{\chi_{\left\{q^{2 k}\right\}}: k \in \mathbb{N}_{0}\right\}$ would be given 
by

$$
\mathcal{D}_{q^{2}}(f)=\sum_{k=0}^{\infty} \frac{f\left(q^{2 k}\right)-f\left(q^{2(k+1)}\right)}{q^{2 k}\left(1-q^{2}\right)} \chi_{\left\{q^{2 k}\right\}},
$$

see for example [KS97, Chapter 2.2]. The extra term $b^{*} a^{*}$ appearing in (2) comes from the geometry of the quantised 2-sphere as it operates between the Hilbert space completions $H_{+}$ and $H_{-}$of the quantised spinor bundles $\mathcal{A}_{1}$ and $\mathcal{A}_{-1}$.

\section{Metric Properties of the Quantised Interval}

In this section we first provide the explicit descriptions of the compact metric spaces $\left(X_{q}, d_{q}\right)$ which encode the compact quantum metric space structure of $\left(I_{q}, L_{D_{q}}\right)$. More precisely, the algebra of Lipschitz functions of the metric space $\left(X_{q}, d_{q}\right)$ must agree with the Lipschitz algebra $\operatorname{Lip}_{D_{q}}\left(S_{q}^{2}\right) \cap I_{q}$ and the two semi-norms must agree, in the sense that $L_{D_{q}}(f(A))=L_{d_{q}}(f)$ whenever $f$ is a Lipschitz function on $\left(X_{q}, d_{q}\right)$. This analysis is separated into the case $q=1$, referred to as the continuum case, and the case $q<1$, referred to as the quantised case.

3.1. The continuum case. We consider the 2 -sphere $S^{2}=\left\{\left(x_{1}, x_{2}, x_{3}\right) \in \mathbb{R}^{3}: x_{1}^{2}+x_{2}^{2}+x_{3}^{2}=\right.$ $1\}$ whereas $S^{3}=\left\{(z, w) \in \mathbb{C}^{2}:|z|^{2}+|w|^{2}=1\right\}$ both equipped with the subspace topology coming from the usual topology on $\mathbb{R}^{3}$ and $\mathbb{C}^{2}$.

In the situation where $q=1$ we have a homeomorphism between the characters of $S U_{q}(2)$ and the 3 -sphere $S^{3}$, which sends $(z, w) \in S^{3} \subset \mathbb{C}^{2}$ to the unique character $\chi_{z, w}$ satisfying that $\chi_{z, w}(a)=z$ and $\chi_{z, w}(b)=w$ (see [Wo87]). Consequently, we can identify $S U_{q}(2)$ with $C\left(S^{3}\right)$ such that $a(z, w)=z$ and $b(z, w)=w$. We may moreover view the 2-sphere $S^{2}$ as the quotient space of $S^{3}$ under the circle action $\lambda \cdot(z, w):=(\lambda \cdot z, \lambda \cdot w)$ and this identification happens via the Hopf-fibration

$$
S^{3} \ni(z, w) \longmapsto\left(2 \operatorname{Re}(z \bar{w}), 2 \operatorname{Im}(z \bar{w}),|z|^{2}-|w|^{2}\right) \in S^{2} .
$$

Since both $A(z, w)=\left(b^{*} b\right)(w)=|w|^{2}$ and $B(z, w)=z \bar{w}$ are invariant under the circle action we may consider them as continuous function on $S^{2}$ and as such they are given by

$$
A\left(x_{1}, x_{2}, x_{3}\right)=\frac{1-x_{3}}{2} \quad \text { and } \quad B\left(x_{1}, x_{2}, x_{3}\right)=\frac{x_{1}+i x_{2}}{2} .
$$

It is now clear that $A$ has range $[0,1]$ and so we have a $*$-isomorphism $C([0,1]) \cong I_{1}$. Let $d_{1}$ be the metric on $[0,1]$ obtained from the standard round metric on $S^{2}$ so that

$d_{1}(s, t):=\inf \left\{d_{S^{2}}\left(\left(x_{1}, x_{2}, 1-2 s\right),\left(y_{1}, y_{2}, 1-2 t\right)\right): x_{1}^{2}+x_{2}^{2}+(1-2 s)^{2}=1=y_{1}^{2}+y_{2}^{2}+(1-2 t)^{2}\right\}$

for all $s, t \in[0,1]$. We record the following elementary result:

Proposition 3.1. The map $\phi:\left[-\frac{\pi}{2}, \frac{\pi}{2}\right] \rightarrow[0,1]$ given by $\phi(t)=\frac{1}{2}+\frac{1}{2} \sin (t)$ is an isometric isomorphism when $\left[-\frac{\pi}{2}, \frac{\pi}{2}\right]$ is equipped with the standard Euclidean metric d and $[0,1]$ is equipped with the metric $d_{1}$. In particular, we have a $*$-isomorphism $\beta: C\left(\left[-\frac{\pi}{2}, \frac{\pi}{2}\right]\right) \rightarrow I_{1}$, $\beta(f)=\left(f \circ \phi^{-1}\right)(A)$, which maps $C_{\mathrm{Lip}}\left(\left[-\frac{\pi}{2}, \frac{\pi}{2}\right]\right)$ onto $I_{1} \cap C_{\mathrm{Lip}}\left(S^{2}\right)$ and satisfies $L_{d_{S^{2}}}(\beta(f))=$ $L_{d}(f)$.

Remark 3.2. For completeness, we note that when $q=1$, the standard Podleś sphere is of course isomorphic to $C\left(S^{2}\right)$. Indeed, the continuous maps corresponding to $A$ and $B$ separate points in $S^{2}$ and the Stone-Weierstrass Theorem then shows that $S_{1}^{2}=C^{*}(1, A, B) \cong C\left(S^{2}\right)$. 
3.2. The quantised case. We will now address the case of a fixed $q \in(0,1)$. We let $X_{q}$ denote the spectrum of $A \in S_{q}^{2}$, and, as we already saw, $X_{q}=\{0\} \cup\left\{q^{2 k}: k \in \mathbb{N}_{0}\right\}$. As explained in the introduction, the Lip-norm $L_{D_{q}}$ gives rise to a metric on the state space of $C^{*}(A, 1) \cong C\left(X_{q}\right)$, which therefore, in particular, determines a metric $d_{q}$ on $X_{q}$ when the latter is viewed as a subset of the state space via point evaluations. The aim of the current section is to find an explicit formula for this metric, and show that the metric spaces $\left(X_{q}, d_{q}\right)$ converge in the Gromov-Hausdorff distance to the Euclidean interval $\left[-\frac{\pi}{2}, \frac{\pi}{2}\right]$ as $q$ tends to 1 .

To this end, we consider the function $\rho_{q}:[-1, \infty) \rightarrow \mathbb{R}$ by

$$
\rho_{q}(x):=\frac{\sqrt{1-q^{2(x+1)}}}{\left(1-q^{2}\right) q^{x}} .
$$

Definition 3.3. Define the metric $d_{q}: X_{q} \times X_{q} \rightarrow[0, \infty)$ by

$$
d_{q}(x, y):= \begin{cases}\sum_{\min \{m, n\}}^{0} \frac{1}{\rho_{q}(k)} & \text { if } x=q^{2 n} \text { and } y=q^{2 m} \text { with } n \neq m \\ \sum_{k=n}^{\infty} \frac{1}{\rho_{q}(k)} & \text { if } x=q^{2 n} \text { and } y=0 \text { or } x=0 \text { and } y=q^{2 n} .\end{cases}
$$

Remark that the series $\sum_{k=0}^{\infty} \frac{1}{\rho_{q}(k)}$ is convergent as can be seen from the estimate

$$
\frac{1}{\rho_{q}(k)}=\frac{q^{k}\left(1-q^{2}\right)}{\sqrt{1-q^{2(k+1)}}} \leqslant q^{k} \quad \text { for all } k \in \mathbb{N}_{0} .
$$

In order to prove Theorem $\mathrm{A}$, we need several lemmas, the first of which shows that the Lipschitz semi-norm on $C\left(X_{q}\right)$ defined by the metric $d_{q}$ and the Lip-norm $L_{D_{q}}$ on $I_{q}$ agree on all finite linear combinations of characteristic functions on $X_{q}$ :

Lemma 3.4. For any $f \in \operatorname{span}_{\mathbb{C}}\left\{\chi_{\left\{q^{2 k}\right\}}: k \in \mathbb{N}_{0}\right\} \subset C\left(X_{q}\right)$, it holds that $f(A) \in \operatorname{Lip}_{D_{q}}\left(S_{q}^{2}\right) \cap$ $I_{q}$. Moreover, we have the identities

$$
L_{D_{q}}(f(A))=\max \left\{\rho_{q}(k) \cdot\left|f\left(q^{2 k}\right)-f\left(q^{2(k+1)}\right)\right|: k \in \mathbb{N}_{0}\right\}=L_{d_{q}}(f) .
$$

In particular, $f$ is also Lipschitz with respect to the metric $d_{q}$.

Note that the maximum is indeed well-defined, since $f$ is non-zero at no more than finitely many elements from $X_{q}$.

Proof. Let $f \in \operatorname{span}_{\mathbb{C}}\left\{\chi_{\left\{q^{2 k}\right\}}: k \in \mathbb{N}_{0}\right\}$ be given. The fact that $f(A) \in \operatorname{Lip}_{D_{q}}\left(S_{q}^{2}\right) \cap I_{q}$ is a consequence of Lemma 2.3. Moreover, from Lemma 2.3 and the defining identities for $S U_{q}(2)$ we obtain that

$$
\begin{aligned}
\partial_{1}(f(A)) \partial_{1}(f(A))^{*} & =A\left(1-q^{2} A\right) \sum_{k=0}^{\infty} \frac{\left|f\left(q^{2 k}\right)-f\left(q^{2(k+1)}\right)\right|^{2}}{q^{4 k}\left(1-q^{2}\right)^{2}} \chi_{\left\{q^{2 k}\right\}}(A) \\
& =\sum_{k=0}^{\infty} \rho_{q}(k)^{2} \cdot\left|f\left(q^{2 k}\right)-f\left(q^{2(k+1)}\right)\right|^{2} \chi_{\left\{q^{2 k}\right\}}(A) .
\end{aligned}
$$

The continuous functional calculus applied to $A \in I_{q}$ then implies that

$$
\left\|\partial_{1}(f(A))\right\|^{2}=\max \left\{\rho_{q}(k) \cdot\left|f\left(q^{2 k}\right)-f\left(q^{2(k+1)}\right)\right|: k \in \mathbb{N}_{0}\right\} .
$$


The identity

$$
L_{D_{q}}(f(A))=\max \left\{\rho_{q}(k) \cdot\left|f\left(q^{2 k}\right)-f\left(q^{2(k+1)}\right)\right|: k \in \mathbb{N}_{0}\right\}
$$

now follows since the formula in (4) implies that $\left\|\partial_{2}(f(A))\right\|=\left\|\partial_{1}(\bar{f}(A))\right\|=\left\|\partial_{1}(f(A))\right\|$.

For the second equality, choose $l \in \mathbb{N}_{0}$ such that

$$
\rho_{q}(l) \cdot\left|f\left(q^{2 l}\right)-f\left(q^{2(l+1)}\right)\right|=\max \left\{\rho_{q}(k) \cdot\left|f\left(q^{2 k}\right)-f\left(q^{2(k+1)}\right)\right|: k \in \mathbb{N}_{0}\right\} .
$$

This choice of $l \in \mathbb{N}_{0}$ implies that

$$
\left|f\left(q^{2 k}\right)-f\left(q^{2(k+1)}\right)\right| \leqslant\left|f\left(q^{2 l}\right)-f\left(q^{2(l+1)}\right)\right| \cdot \frac{\rho_{q}(l)}{\rho_{q}(k)}
$$

for all $k \in \mathbb{N}_{0}$. Thus, for every $m<n$ we may now estimate as follows:

$$
\begin{aligned}
\left|f\left(q^{2 m}\right)-f\left(q^{2 n}\right)\right| & \leqslant \sum_{k=m}^{n-1}\left|f\left(q^{2 k}\right)-f\left(q^{2(k+1)}\right)\right| \leqslant \sum_{k=m}^{n-1}\left|f\left(q^{2 l}\right)-f\left(q^{2(l+1)}\right)\right| \cdot \frac{\rho_{q}(l)}{\rho_{q}(k)} \\
& =\left|f\left(q^{2 l}\right)-f\left(q^{2(l+1)}\right)\right| \cdot \rho_{q}(l) \cdot d_{q}\left(q^{2 m}, q^{2 n}\right) .
\end{aligned}
$$

This shows that $f: X_{q} \rightarrow \mathbb{C}$ is Lipschitz with $L_{d_{q}}(f) \leqslant L_{D_{q}}(f(A))$. The fact that equality is achieved is then a consequence of Definition 3.3. Indeed, we obtain that

$$
L_{D_{q}}(f(A))=\left|f\left(q^{2 l}\right)-f\left(q^{2(l+1)}\right)\right| \cdot \rho_{q}(l)=\frac{\left|f\left(q^{2 l}\right)-f\left(q^{2(l+1)}\right)\right|}{d_{q}\left(q^{2 l}, q^{2(l+1)}\right)} \leqslant L_{d_{q}}(f) .
$$

The next lemma computes the Lipschitz semi-norms of general continuous functions on $X_{q}$ and provides information on the behaviour of the Lipschitz constants of a particularly interesting approximation.

Lemma 3.5. For any $f \in C\left(X_{q}\right)$ one has

$$
L_{d_{q}}(f)=\sup \left\{\left|f\left(q^{2 k}\right)-f\left(q^{2(k+1)}\right)\right| \cdot \rho_{q}(k): k \in \mathbb{N}_{0}\right\}
$$

Moreover, if $f(0)=0$ and $f$ is Lipschitz with respect to the metric $d_{q}$, then the sequence $\left\{L_{d_{q}}\left(f \cdot \chi_{\left\{q^{2 k}: k \leqslant n\right\}}\right)\right\}_{n=0}^{\infty}$ is bounded.

Proof. We first notice that Definition 3.3 implies the inequality

$$
\sup \left\{\left|f\left(q^{2 k}\right)-f\left(q^{2(k+1)}\right)\right| \cdot \rho_{q}(k): k \in \mathbb{N}_{0}\right\} \leqslant L_{d_{q}}(f)
$$

(see also the proof of Lemma 3.4 for more details).

We then claim that

$$
\frac{|f(x)-f(y)|}{d_{q}(x, y)} \leqslant \sup \left\{\left|f\left(q^{2 k}\right)-f\left(q^{2(k+1)}\right)\right| \cdot \rho_{q}(k): k \in \mathbb{N}_{0}\right\}
$$

whenever $x, y \in X_{q} \backslash\{0\}$ satisfy $x \neq y$. We have to be careful at this point since the inequality in (6) is not an immediate consequence of Definition 3.3 : the right hand side of our inequality only uses successive elements as exponents (i.e. $k$ and $k+1$ ) whereas $x=q^{2 n}$ and $y=q^{2 m}$ for some $n, m \in \mathbb{N}_{0}$ without any further constraints (except for $n \neq m$ ). The inequality in (6) does however follow by an application of Lemma 3.4 to a suitable restriction of $f$.

Thus, to establish the claimed identity, it only remains to be shown that the supremum in (6) is still an upper bound when $x=q^{2 n}$ for some $n \in \mathbb{N}_{0}$ and $y=0$. However, this follows immediately from the estimate in (6) together with continuity of the function $f$ and the metric $d_{q}$ 
For the second part, we assume that $f$ is Lipschitz and that $f(0)=0$. By Lemma 3.4 it suffices to show that the sequence $\left\{\left|f\left(q^{2 n}\right)\right| \cdot \rho_{q}(n)\right\}_{n=0}^{\infty}$ is bounded. To this end, we first note that since $f$ is Lipschitz we may find a constant $C$ such that $\left|f\left(q^{2 n}\right)\right| \leqslant C \cdot d_{q}\left(q^{2 n}, 0\right)$ for all $n \in \mathbb{N}_{0}$. It follows that

$$
\rho_{q}(n) \cdot\left|f\left(q^{2 n}\right)\right| \leqslant C \cdot \sum_{k=n}^{\infty} \frac{\rho_{q}(n)}{\rho_{q}(k)}=C \cdot \sum_{k=0}^{\infty} q^{k} \frac{\sqrt{1-q^{2(n+1)}}}{\sqrt{1-q^{2(k+n+1)}}} \leqslant C \cdot \sum_{k=0}^{\infty} q^{k}=\frac{C}{1-q}
$$

for all $n \in \mathbb{N}_{0}$. This ends the proof of the lemma.

The metric $d_{q}: X_{q} \times X_{q} \rightarrow[0, \infty)$ yields a Lipschitz algebra $C_{\text {Lip }}\left(X_{q}\right) \subset C\left(X_{q}\right)$ and the semi-norm $L_{D_{q}}: I_{q} \rightarrow[0, \infty]$ yields an a priori different Lipschitz algebra $\operatorname{Lip}_{D_{q}}\left(I_{q}\right) \subset I_{q}$. The Lipschitz algebras $C_{\text {Lip }}\left(X_{q}\right)$ and $\operatorname{Lip}_{D_{q}}\left(I_{q}\right)$ agree with the domains of the semi-norms $L_{d_{q}}$ and $L_{D_{q}}$, respectively (recall that the domain consists of the elements where a semi-norm is finite). Moreover, the two unital commutative $C^{*}$-algebras $C\left(X_{q}\right)$ and $I_{q}$ are related by the *-isomorphisms $f \mapsto f(A)$. We are going to show that the $*$-isomorphism $f \mapsto f(A)$ restricts to a $*$-isomorphism $C_{\text {Lip }}\left(X_{q}\right) \rightarrow \operatorname{Lip}_{D_{q}}\left(I_{q}\right)$ which is moreover isometric with respect to the semi-norms $L_{d_{q}}$ and $L_{D_{q}}$.

Suppressing the identification $C\left(X_{q}\right) \cong I_{q}$ we have by now proved that the two semi-norms $L_{d_{q}}$ and $L_{D_{q}}$ agree on finite linear combinations of the indicator functions $\chi_{\left\{q^{2 k}\right\}}, k \in \mathbb{N}_{0}$ (Lemma 3.4) and we have moreover succeeded in computing the semi-norm $L_{d_{q}}: C\left(X_{q}\right) \rightarrow$ $[0, \infty]$ (Lemma 3.5) .

The passage from finite linear combinations of indicator functions to general Lipschitz elements is however quite subtle. To explain a bit what the subtle point is, we let $\mathcal{J}_{q} \subset I_{q}$ denote the smallest unital $*$-subalgebra containing all the projections $\chi_{\left\{q^{2 k}\right\}}(A)$. Then even though $\mathcal{J}_{q} \subset I_{q}$ is norm-dense and the derivation $\partial: \operatorname{Lip}_{D_{q}}\left(I_{q}\right) \rightarrow B\left(H_{+} \oplus H_{-}\right)$is closed, it is not true that $\operatorname{Lip}_{D_{q}}\left(I_{q}\right)$ can be recovered by taking the closure of the restriction $\partial: \mathcal{J}_{q} \rightarrow B\left(H_{+} \oplus H_{-}\right)$. In particular, for a general element $f(A)=f(0)+\sum_{k=0}^{\infty} f\left(q^{2 k}-f(0)\right) \cdot \chi_{\left\{q^{2 k}\right\}}(A) \in \operatorname{Lip}_{D_{q}}\left(I_{q}\right)$ we cannot a priori compute $\partial(f(A)) \in B\left(H_{+} \oplus H_{-}\right)$by using Lemma 2.3 and applying the derivation $\partial$ term by term.

After these clarifications we are ready to state and prove the first main result of this section:

Theorem 3.1. The Lip-algebra of $I_{q}$ associated with the Dabrowski-Sitarz spectral triple $\left(S_{q}^{2}, H_{+} \oplus H_{-}, D_{q}\right)$ agrees with $\left\{f(A): f \in C_{\text {Lip }}\left(X_{q}\right)\right\}$, and for $f \in C_{\text {Lip }}\left(X_{q}\right)$, we have $L_{D_{q}}(f(A))=L_{d_{q}}(f)$.

Proof. Let $f \in C\left(X_{q}\right)$ be given.

Suppose first that $L_{D_{q}}(f(A))<\infty$. For each $n \in \mathbb{N}_{0}$ we define the projection $Q_{n}:=$ $\sum_{k=0}^{n} \chi_{\left\{q^{2 k}\right\}}(A)$. Since $\partial_{1}$ is a derivation, we obtain from Lemma 2.3 that

$$
\begin{aligned}
\partial_{1}(f(A)) Q_{n} & =\partial_{1}\left(f(A) Q_{n}\right)-f(A) \partial_{1}\left(Q_{n}\right) \\
& =\sum_{k=0}^{n-1}\left(f\left(q^{2 k}\right)-f\left(q^{2(k+1)}\right)\right) \frac{1}{q^{2 k}\left(1-q^{2}\right)} \chi_{\left\{q^{2 k}\right\}}(A) \cdot b^{*} a^{*} .
\end{aligned}
$$

Following the proof of Lemma 3.4 we then get that

$$
\left\|\partial_{1}(f(A)) Q_{n}\right\|=\max \left\{\left|f\left(q^{2 k}\right)-f\left(q^{2(k+1)}\right)\right| \cdot \rho_{q}(k): k \in\{0,1, \ldots, n-1\}\right\}
$$


and hence (using that $Q_{n}$ is an orthogonal projection) we obtain the estimate

$$
\begin{aligned}
\sup \left\{\left|f\left(q^{2 k}\right)-f\left(q^{2(k+1)}\right)\right| \cdot \rho_{q}(k): k \in \mathbb{N}_{0}\right\} & =\sup \left\{\left\|\partial_{1}(f(A)) Q_{n}\right\|: n \in \mathbb{N}_{0}\right\} \\
& \leqslant\left\|\partial_{1}(f(A))\right\| .
\end{aligned}
$$

By Lemma 3.5 this shows that $f$ is Lipschitz with respect to the metric $d_{q}$ and that

$$
L_{d_{q}}(f) \leqslant\left\|\partial_{1}(f(A))\right\| .
$$

To prove that equality holds, we observe that by [Ti08, Theorem 6.2.17],

$$
h\left(Q_{n}\right)=\left(1-q^{2}\right) \sum_{k=0}^{n} q^{2 k} \underset{n \rightarrow \infty}{\longrightarrow} 1,
$$

where $h$ denotes the Haar state on $S U_{q}(2)$. Since $h$ is faithful and $\left\{Q_{n}\right\}_{n=0}^{\infty}$ is an increasing sequence of projections, $Q_{n}$ converges to the identity in the strong operator topology on $B\left(L^{2}\left(S U_{q}(2)\right)\right)$, and hence also on $B\left(H_{+}\right)$. It now follows from (8) and Lemma 3.5 that for any $\xi$ in the unit ball of $H_{+}$, we have

$$
\begin{aligned}
\left\|\partial_{1}(f(A)) \xi\right\| & =\lim _{n \rightarrow \infty}\left\|\partial_{1}(f(A)) Q_{n} \xi\right\| \leqslant \sup \left\{\left\|\partial_{1}(f(A)) Q_{n}\right\|: n \in \mathbb{N}_{0}\right\} \\
& =L_{d_{q}}(f) .
\end{aligned}
$$

and hence that $\left\|\partial_{1}(f(A))\right\|=L_{d_{q}}(f)$. Since we moreover have the identities

$$
\left\|\partial_{2}(f(A))\right\|=\left\|\partial_{1}(\bar{f}(A))\right\|=L_{d_{q}}(f)
$$

we may conclude that $L_{D_{q}}(f(A))=L_{d_{q}}(f)$.

Suppose next that $f \in C\left(X_{q}\right)$ is Lipschitz with respect to the metric $d_{q}$. Since subtracting a constant changes neither the Lipschitz constant of $f$ nor $L_{D_{q}}(f(A))$, we may, without loss of generality, assume that $f(0)=0$. For each $n \in \mathbb{N}_{0}$ define the function $f_{n}:=f \cdot \chi_{\left\{q^{2 k}: k \leqslant n\right\}}$. By Lemma 3.5, the sequence $\left\{L_{d_{q}}\left(f_{n}\right)\right\}_{n=0}^{\infty}$ is then bounded and moreover $f_{n}(A)$ converges to $f(A)$ in operator norm.

Hence, since $L_{D_{q}}\left(f_{n}(A)\right)=L_{d_{q}}\left(f_{n}\right)$ by Lemma 3.4, we obtain by lower semi-continuity of $L_{D_{q}}: I_{q} \rightarrow[0, \infty]$ that

$$
L_{D_{q}}(f(A)) \leqslant \sup \left\{L_{D_{q}}\left(f_{n}(A)\right): n \in \mathbb{N}_{0}\right\}<\infty .
$$

This shows that $f(A) \in \operatorname{Lip}_{D_{q}}\left(I_{q}\right)$ and this ends the proof of the theorem.

Theorem A now follows easily:

Proof of Theorem $A$. The metric $d_{q}^{\prime}$ on $X_{q}$ induced by $L_{D_{q}}$ is by definition given by

$$
d_{q}^{\prime}(x, y):=\sup \left\{|f(x)-f(y)|: f \in C\left(X_{q}\right), L_{D_{q}}(f(A)) \leqslant 1\right\} .
$$

However, by Theorem 3.1 we have

$$
\begin{aligned}
d_{q}(x, y) & =\sup \left\{|f(x)-f(y)|: f \in C\left(X_{q}\right), L_{d_{q}}(f) \leqslant 1\right\} \\
& =\sup \left\{|f(x)-f(y)|: f \in C\left(X_{q}\right), L_{D_{q}}(f(A)) \leqslant 1\right\},
\end{aligned}
$$

and hence the two metrics agree.

In the following, we will consider the behaviour of $\left(X_{q}, d_{q}\right)$ with respect to the GromovHausdorff metric, and provide a proof of Theorem B. To this end, we first establish a preliminary result about the diameter of $X_{q}$ :

Lemma 3.6. It holds that $\lim _{q \rightarrow 1} d_{q}(0,1)=\pi$. 
Proof. Observe that the function $\frac{1}{\rho_{q}}: x \mapsto\left(1-q^{2}\right) \frac{q^{x}}{\sqrt{1-q^{2(x+1)}}}$ is positive and decreasing on $(-1, \infty)$. This yields the estimates

$$
\int_{1}^{\infty} \frac{1}{\rho_{q}(x)} d x \leqslant \sum_{k=0}^{\infty} \frac{1}{\rho_{q}(k)} \leqslant \int_{0}^{\infty} \frac{1}{\rho_{q}(x)} d x .
$$

Furthermore, it can be verified that $F(x):=\frac{1-q^{2}}{q \ln (q)} \arcsin \left(q^{x+1}\right)$ is an antiderivative of $\frac{1}{\rho_{q}(x)}$ and $\lim _{x \rightarrow \infty} F(x)=0$. We therefore obtain the inequalities

$$
-\frac{1-q^{2}}{q \ln (q)} \arcsin \left(q^{2}\right) \leqslant d_{q}(0,1) \leqslant-\frac{1-q^{2}}{q \ln (q)} \arcsin (q) .
$$

Since $\lim _{q \rightarrow 1} \frac{1-q^{2}}{q \ln (q)}=-2$ and $\arcsin (1)=\frac{\pi}{2}$ we may conclude that $\lim _{q \rightarrow 1} d_{q}(0,1)=\pi$.

Proof of Theorem $\mathbb{B}$. For each $q \in(0,1)$, we consider the isometric embedding $\iota_{q}: X_{q} \rightarrow \mathbb{R}$ given by $\iota_{q}(x)=d_{q}(1, x)-\frac{\pi}{2}$.

We start by proving continuity at a fixed $q_{0} \in(0,1)$. Let $\varepsilon>0$ be given. Choose a $\delta_{0}>0$ such that $J:=\left[q_{0}-\delta_{0}, q_{0}+\delta_{0}\right] \subset(0,1)$. From the estimate in (3) we obtain that

$$
\sum_{k=0}^{\infty} \sup \left\{\frac{1}{\rho_{q}(k)}: q \in J\right\} \leqslant \sum_{k=0}^{\infty} \sup \left\{q^{k} \sqrt{1-q^{2}}: q \in J\right\} \leqslant \sum_{k=0}^{\infty}\left(q_{0}+\delta_{0}\right)^{k}<\infty .
$$

We may therefore choose an $n_{0} \in \mathbb{N}_{0}$ such that

$$
\sum_{k=n_{0}}^{\infty} \frac{1}{\rho_{q}(k)}<\frac{\varepsilon}{3}
$$

for all $q \in J=\left[q_{0}-\delta_{0}, q_{0}+\delta_{0}\right]$. Now, for each $k \in \mathbb{N}_{0}$, the function $q \mapsto \sum_{k=0}^{n_{0}-1} \frac{1}{\rho_{q}(k)}$ is continuous and we may thus choose a $\delta \in\left(0, \delta_{0}\right)$ such that

$$
\left|\sum_{k=0}^{m-1} \frac{1}{\rho_{q}(k)}-\sum_{k=0}^{m-1} \frac{1}{\rho_{q_{0}}(k)}\right|<\frac{\varepsilon}{3}
$$

for all $m \in\left\{1, \ldots, n_{0}\right\}$ and all $q \in\left(q_{0}-\delta, q_{0}+\delta\right)$.

Let now $q \in\left(q_{0}-\delta, q_{0}+\delta\right) \subset J$ be given. It then follows immediately from (11) that

$$
\left|\iota_{q}\left(q^{2 m}\right)-\iota_{q_{0}}\left(q_{0}^{2 m}\right)\right|<\frac{\varepsilon}{3}<\varepsilon
$$

for all $m \in\left\{1, \ldots, n_{0}\right\}$. Moreover, for $m>n_{0}$ we apply (10) and (111) to estimate that

$$
\begin{aligned}
\left|\iota_{q}\left(q^{2 m}\right)-\iota_{q_{0}}\left(q_{0}^{2 m}\right)\right| & =\left|\sum_{k=0}^{n_{0}-1} \frac{1}{\rho_{q}(k)}+\sum_{k=n_{0}}^{m-1} \frac{1}{\rho_{q}(k)}-\sum_{k=0}^{n_{0}-1} \frac{1}{\rho_{q_{0}}(k)}-\sum_{k=n_{0}}^{m-1} \frac{1}{\rho_{q_{0}}(k)}\right| \\
& \leqslant\left|\iota_{q}\left(q^{2 n_{0}}\right)-\iota_{q_{0}}\left(q_{0}^{2 n_{0}}\right)\right|+\sum_{k=n_{0}}^{\infty} \frac{1}{\rho_{q}(k)}+\sum_{k=n_{0}}^{\infty} \frac{1}{\rho_{q_{0}}(k)} \\
& <\varepsilon .
\end{aligned}
$$

A similar argument also shows that $\left|\iota_{q}(0)-\iota_{q_{0}}(0)\right|<\varepsilon$. We conclude that

$$
\operatorname{dist}_{H}\left(\iota_{q}\left(X_{q}\right), \iota_{q_{0}}\left(X_{q_{0}}\right)\right) \leqslant \varepsilon
$$

and hence that $(0,1) \ni q \mapsto\left(X_{q}, d_{q}\right)$ varies continuously in Gromov-Hausdorff distance. 
For convergence, it suffices to show that the Hausdorff distance between $\iota_{q}\left(X_{q}\right)$ and $\left[-\frac{\pi}{2}, \frac{\pi}{2}\right]$ converges to 0 as $q \rightarrow 1$. To this end, let $\varepsilon>0$ be arbitrary. By Lemma 3.6, we may find a $q_{1} \in(0,1)$ such that for any $q \in\left(q_{1}, 1\right)$, we have $\left|\iota_{q}(0)-\frac{\pi}{2}\right|<\varepsilon$. Moreover, since $-\frac{\pi}{2} \leqslant \iota_{q}(x) \leqslant \iota_{q}(0)$ for all $x \in X_{q}$, it follows that for every $x \in X_{q}$ there exists a $y \in\left[-\frac{\pi}{2}, \frac{\pi}{2}\right]$ with $\left|\iota_{q}(x)-y\right|<\varepsilon$. It remains to be shown that we can find a $q_{2} \in(0,1)$ such that given any $y \in\left[-\frac{\pi}{2}, \frac{\pi}{2}\right]$ and any $q \in\left(q_{2}, 1\right)$, we can find $x \in X_{q}$ such that $\left|y-\iota_{q}(x)\right|<\varepsilon$. Since $\frac{1}{\rho_{q}(0)}=\sqrt{1-q^{2}} \underset{q \rightarrow 1}{\longrightarrow} 0$ and $d_{q}(0,1) \underset{q \rightarrow 1}{\longrightarrow} \pi$ by Lemma 3.6 we can find a $q_{2} \in(0,1)$ such that $\frac{1}{\rho_{q}(0)}<\varepsilon$ and $\left|\iota_{q}(0)-\frac{\pi}{2}\right|<\frac{\varepsilon}{2}$ for all $q \in\left(q_{2}, 1\right)$. Let now $q \in\left(q_{2}, 1\right)$ be given. It follows that $\left|y-\iota_{q}(0)\right|<\varepsilon$ for $y \in\left(\frac{\pi}{2}-\frac{\varepsilon}{2}, \frac{\pi}{2}\right]$. On the other hand, we may for each $y \in\left[-\frac{\pi}{2}, \frac{\pi}{2}-\frac{\varepsilon}{2}\right]$ find an $n \in \mathbb{N}_{0}$ such that $y \in\left[\iota_{q}\left(q^{2 n}\right), \iota_{q}\left(q^{2(n+1)}\right)\right]$ and consequently

$$
\left|y-\iota_{q}\left(q^{2 n}\right)\right| \leqslant\left|\iota_{q}\left(q^{2 n}\right)-\iota_{q}\left(q^{2(n+1)}\right)\right|=\frac{1}{\rho_{q}(n)} \leqslant \frac{1}{\rho_{q}(0)}<\varepsilon .
$$

Remark 3.7. As stated in the introduction, Theorem B also applies if we replace the classical Gromov-Hausdorff distance with respectively the quantum Gromov-Hausdorff distance of Rieffel [Ri04] or Latrémolière's propinquity. To see this, note that by [La16, Corollary 6.4] the former is dominated by two times the latter and by [La16. Theorem 6.6], propinquity is dominated by the classical Gromov-Hausdorff distance on the class of compact metric spaces, and hence the convergence and continuity are also obtained for these distances.

\section{REFERENCES}

[AK18] Konrad Aguilar and Jens Kaad. The Podleś Sphere as a Spectral Metric Space. J. Geom. Phys. 133 (2018), 260-278.

[AKK21] Konrad Aguilar, Jens Kaad and David Kyed. The Podles spheres converge to the sphere. Preprint. arXiv:2102.12761

[Co89] Alain Connes. Compact metric spaces, Fredholm modules, and hyperfiniteness. Ergodic Theory Dynam. Systems, 9 (1989), no. 2, 207-220.

[DS03] Ludwik Dąbrowski and Andrzej Sitarz. Dirac operator on the standard Podleś quantum sphere. Noncommutative geometry and quantum groups (Warsaw, 2001), 49-58, Banach Center Publ., 61, Polish Acad. Sci. Inst. Math., Warsaw, 2003.

[KS97] Anatoli Klimyk and Konrad Schmüdgen. Quantum Groups and their Representations. Texts and Monographs in Physics, Springer-Verlag, Berlin, 1997.

[La16] Frédéric Latrémolière. The quantum Gromov-Hausdorff propinquity. Trans. Amer. Math. Soc. 368 (2016), no. 1, 365-411.

[NeTu05] Sergey Neshveyev and Lars Tuset. A local index formula for the quantum sphere. Commun. Math. Phys. 254 (2005), no. 2, 323-341.

[Po87] Piotr Podleś. Quantum Spheres. Lett. Math. Phys. 14 (1987), no. 3, 193-202.

[Ri98] Marc Rieffel. Metrics on states from actions of compact groups. Doc. Math. 3 (1998), 215-229.

[Ri99] Marc Rieffel. Metrics on state spaces. Doc. Math. 4 (1999), 559-600.

[Ri04] Marc Rieffel. Gromov-Hausdorff Distance for Quantum Metric Spaces. Mem. Amer. Math. Soc. 168 (2004), no. 796, 1-65.

[Ri05] Marc Rieffel. Compact quantum metric spaces. Operator Algebras, Quantization, and Noncommutative Geometry, 315-330, Contemp. Math., 365, Amer. Math. Soc., Providence, RI, 2004.

[Ti08] Thomas Timmermann. An invitation to quantum groups and duality. From Hopf algebras to multiplicative unitaries and beyond. EMS Textbooks in Mathematics. European Mathematical Society (EMS), Zürich, 2008.

[Wo87] Stanisław. L. Woronowicz. Twisted $S U(2)$ group. An example of a noncommutative differential calculus. Publ. Red. Inst. Math. Sci. 23 (1987), no. 1, 117-181. 
Thomas Gotfredsen, Department of Mathematics and Computer Science, University of Southern Denmark, Campusvej 55, DK-5230 Odense M, Denmark

Email address: thgot@imada.sdu.dk

Jens Kaad, Department of Mathematics and Computer Science, University of Southern Denmark, Campusvej 55, DK-5230 Odense M, Denmark

Email address: kaad@imada.sdu.dk

David Kyed, Department of Mathematics and Computer Science, University of Southern Denmark, Campusvej 55, DK-5230 Odense M, Denmark

Email address: dkyed@imada.sdu.dk 